Linear and Multilinear Algebra, 1993, Vol. 34, pp. 3-19

Reprints available directly from the publisher

Photocopying permitted by license only

(C) 1993 Gordon and Breach Science Publishers S.A.

Printed in the United States of America

\title{
Ranks of Zero Patterns and Sign Patterns*
}

\author{
DANIEL HERSHKOWITZ** \\ Mathematics Department, Technion-Israel Institute of Technology, Haifa 32000, Israel \\ HANS SCHNEIDER $\dagger$ \\ Mathematics Department, University of Wisconsin-Madison, Madison, WI 53706
}

(Received March 19, 1991; in final form March 25, 1992)

Let $\mathbf{F}$ be a field with at least three elements. Zero patterns $P$ such that all matrices over $F$ with pattern $P$ have the same rank are characterized. Similar results are proven for sign patterns. These results are applied to answering two open questions on conditions for formal nonsingularity of a pattern $P$, as well as to proving a sufficient condition on $P$ such that all matrices over $F$ with pattern $P$ have the same height characteristic.

\section{INTRODUCTION}

The height characteristic of a square matrix $A$ is defined to be the sequence of differences of nullities of powers of $A$. As such, the height characteristic of $A$ is determined by the ranks of powers of $A$. The discussion in this paper relates to the case of combinatorially determined height characteristic, that is when the height characteristic of $A$ is determined by the zero pattern or the sign pattern of $A$. In particular, we discuss ranks of rectangular matrices whose zero pattern or sign pattern is given. Our results are highly graph theoretic, using matchings and constrained matchings in bipartite graphs as well as in directed graphs.

We now describe the paper in more detail.

Most of our notation and definitions are given in Section 2. In Section 3 we study ranks of matrices whose zero pattern is given. Among others, we prove that given an $m \times n$ pattern matrix $P$ and a field $\mathbf{F}$ with at least three elements, every matrix over $\mathbf{F}$ with pattern $P$ has rank $r$ if and only if the bipartite graph $B(P)$ of $P$ has no $t$-matching for $t>r$, and there exists at least one constrained $r$-matching in $B(P)$.

A square matrix $A$ is said to have property $C$ if in every row and every column of $A$ there exists just one element such that both this element and its cofactor are nonzero. A square matrix $A$ is said to have property $D$ if it is permutationally equiv-

\footnotetext{
*Dedicated to Henryk Minc on the occasion of his 70th birthday.

This research was supported by a joint grant No. 90-00434 from the United States-Israel Binational Science Foundation, Jerusalem, Israel.

**The research of this author was supported by the New York Metropolitan Research Fund.

tThe research of this author was supported in part by NSF grants DMS-8521521, DMS-8901445, and EMS-8718971, and by the International Scientific Research Program, the Ministry of Education, Science and Culture, Japan.
} 
alent to a matrix $A^{\prime}$ such that the proper principal minors of $A^{\prime}$ are nonzero, and the product of every two complementary non-principal minors of $A^{\prime}$ is zero. In a paper by Culik [5], the author proves two theorems, characterizing formally nonsingular pattern matrices, that is patterns $P$ such that every matrix with pattern $P$ is nonsingular. Among others, he proves that every matrix over a field $\mathbf{F}$ with at least 3 elements with pattern $P$ is nonsingular if and only if every matrix $A$ over $\mathbf{F}$ with pattern $P$ has property $C$. It is also proven there that another equivalent condition is: $P$ is permutationally equivalent to a pattern matrix $P^{\prime}$ for which we have: the proper principal subpatterns of $P^{\prime}$ are all formally nonsingular over $\mathrm{F}$, and every matrix over $\mathbf{F}$ with pattern $P^{\prime}$ has property $D$. The author poses the questions whether these conditions can be weakened to have them required on some matrix rather than on every matrix. In Section 4 we answer these questions. We show, by means of an example, that one of the conditions cannot be weakened as suggested, while the other one can be replaced by a statement that is even weaker than the suggested one.

In Section 5 we carry on a discussion similar to the one in Section 3, investigating sign patterns. Our main result here asserts that every matrix with an $m \times n$ sign pattern $P$ has rank $r$ if and only if $P$ is permutationally equivalent to a pattern

$$
\left(\begin{array}{cc}
P_{11} & P_{12} \\
P_{21} & 0
\end{array}\right), \quad P_{11} \text { is } k \times(r-k), \quad 0 \leq k \leq r,
$$

where every nonzero $(+,-, 0)$-row scaling of $P_{12}$ has a unisign column, and every $(+,-, 0)$-column scaling of $P_{21}$ has a unisign row.

In Section 6 we apply the results of Sections 3 and 5 to the question of characterizing square patterns $P$ such that for every positive integer $k$ and for all matrices $A$ with pattern $P$, the matrices $A^{k}$ have the same rank. This is essentially the problem of characterizing graphs with combinatorially determined height characteristic. This problem is, in general, open, and has been discussed in a few papers, e.g. [3] and [4]. Here we prove a sufficient condition on $P$ such that all matrices over a field of at least three elements $\mathbf{F}$ with pattern $P$ have the same height characteristic. Our assertion yields that whenever the graph $G(P)$ of $P$ is strongly triangular, all matrices over $\mathbf{F}$ with pattern $P$ have the same height characteristic.

\section{NOTATION AND DEFINITIONS}

This section contains most of the notation and definitions used in this paper. Some are given in the next sections.

Notation 2.1 We denote:

$|S|$ - the cardinality of a set $S$.

$\langle n\rangle$ - the set $\{1,2, \ldots, n\}$.

Notation 2.2 Let $A$ be an $m \times n$ matrix and let $\alpha$ and $\beta$ be subsets of $\langle m\rangle$ and $\langle n\rangle$ respectively. We denote: 
$A[\alpha \mid \beta]$ - the submatrix of $A$ whose rows are indexed by $\alpha$ and whose columns are indexed by $\beta$ in their natural order. $\alpha, \beta \neq \varnothing$.

$$
\begin{aligned}
& A(\alpha \mid \beta)=A[\langle m\rangle \backslash \alpha \mid\langle n\rangle \backslash \beta] . \quad \alpha \neq\langle m\rangle, \quad \beta \neq\langle n\rangle . \\
& A[\alpha \mid \beta)=A[\alpha \mid\langle n\rangle \backslash \beta] . \quad \alpha \neq \emptyset, \quad \beta \neq\langle n\rangle . \\
& A(\alpha \mid \beta]=A[\langle m\rangle \backslash \alpha \mid \beta] . \quad \alpha \neq\langle m\rangle, \quad \beta \neq \emptyset .
\end{aligned}
$$

DEFINTTION 2.3 Two matrices $A$ and $B$ are said to be permutationally equivalent if there exist permutation matrices $P_{1}$ and $P_{2}$ such that $A=P_{1} B P_{2}$.

DEFINTTION 2.4 Let $B$ be a bipartite graph. A set of $t$ edges $\left[i_{1}, j_{1}\right], \ldots,\left[i_{t}, j_{t}\right]$ in $B$ is said to be a $t$-matching (between $\left\{i_{1}, \ldots, i_{t}\right\}$ and $\left\{j_{1}, \ldots, j_{t}\right\}$ ) if $i_{1}, \ldots, i_{t}$ are distinct and $j_{1}, \ldots, j_{t}$ are distinct. Such a $t$-matching is said to be a contrained $t$-matching if it is the only $t$-matching in $B$ between $\left\{i_{1}, \ldots, i_{t}\right\}$ and $\left\{j_{1}, \ldots, j_{t}\right\}$.

A different formulation for $t$-matchings in the case that the two vertex sets in the bipartite graph $B$ have the same cardinality (in which case $B$ can be written as a simple directed graph) is given in Section 6.

DEFINTTION 2.5 Let $A$ be an $m \times n$ matrix. The bipartite graph $B(A)$ of $A$ is defined to be the bipartite graph with the sets $\{1, \ldots, m\}$ and $\left\{1^{\prime}, \ldots n^{\prime}\right\}$ of nodes, and where $\left[i, j^{\prime}\right]$ is an edge in $B(A)$ whenever $a_{i j} \neq 0$.

Convention 2.6 We shall use the set $\{1, \ldots, n\}$ for the second set $\left\{1^{\prime}, \ldots n^{\prime}\right\}$ of nodes in Definition 2.5 where no confusion should arise.

\section{DEFINITION 2.7}

(i) $A$ matrix is said to be a (zero) pattern matrix if each element of $A$ is either a zero or a star.

(ii) $A$ pattern of a matrix $A$ over an arbitrary field is the pattern matrix obtained by replacing every nonzero entry of $A$ by a star.

(iii) $A$ square pattern matrix is said to be formally singular [nonsingular] over a field $\mathbf{F}$ if every matrix over $\mathbf{F}$ with pattern $P$ is singular [nonsingular].

\section{DEFINITION 2.8}

(i) A matrix is said to be a sign pattern matrix if each element of $A$ is either a zero or a "+" or a "-".

(ii) $A$ sign pattern of a real matrix $A$ is the sign pattern matrix obtained by replacing every nonzero entry of $A$ by its sign.

(iii) $A$ sign pattern matrix is said to be a nonnegative sign pattern matrix if it has no "-" elements.

(iv) A square sign pattern matrix is said to be formally singular [nonsingular] if every real matrix with sign pattern $P$ is singular [nonsingular].

DEFINITION 2.9 Let $P$ be a sign matrix.

(i) A multiplication of a row [column] of $P$ by " + " is leaving that row [column] as it is. A multiplication of a row [column] of $P$ by "-" is changing all the 
signs of the nonzero elements in that row [column]. A multiplication of a row [column] of $P$ by zero is replacing that row [column] by a zero row [column].

(ii) $A(+,-, 0)$-row [column] scaling of $P$ is a sign pattern matrix that is obtained by multiplying each row [column] of $P$ by either "+" or "- " or zero.

(iii) $A(+,-, 0)$-row [column] scaling of $P$ is said to be a nonzero scaling if at least one row [column] of $P$ is not multiplied by zero.

DEFINITION 2.10 A row [column] of a sign pattern matrix is said to be unisign if all the nonzero elements in that row [column] have the same sign.

\section{DEFINITION 2.11}

(i) Let $S$ be a set of $n$-vectors. The support of $S$ is defined to be the subset of $\langle n\rangle$ of all $i$ such that there exists at least one vector in $S$ with a nonzero ith component.

(ii) $A$ set $S$ of $n$-vectors is said to be combinatorially independent if it does not contain the zero vector and every two non-empty disjoint subsets of $S$ have different supports.

\section{DEFINITION 2.12 Let $G$ be a directed graph.}

(i) $A$ path in $G$ is a sequence of distinct vertices $\left(i_{1}, \ldots, i_{t}\right)$ such that $\left(i_{k}, i_{k+1}\right)$ is an arc in $G, k \in\langle t-1\rangle$. A path may consist of just one vertex.

(ii) Two paths in $G$ are said to be disjoint if they have no common vertex.

(iii) Let $M$ be a set of vertices in $G$. A set $\prod$ of disjoint paths in $G$ is called a path covering for $M$ if every vertex of $M$ belongs to exactly one path in $\Pi$. Observe that a path covering for $M$ may cover also vertices that are not in $M$.

DEFINITION $2.13 A$ vertex $i$ in a directed graph is said to be singular if the loop (i.i) is not in $G$. Otherwise the vertex is said to be nonsingular.

DEFINITION 2.14 (see [9])

(i) The length $|\pi|$ of a path $\pi$ in $G$ is the number of vertices in $\pi$.

(ii) The singular length $\left|\pi^{s}\right|$ of a path $\pi$ in $G$ is the number of singular vertices in $\pi$.

(iii) The kth singular norm of a path $\pi$ is defined to be $\left|\prod^{s}\right|_{k}=\min \left\{\left|\pi^{s}\right|, k\right\}$.

(iv) Let $\Pi=\left\{\pi_{1}, \ldots, \pi_{t}\right\}$ be a set of disjoint paths in $G$. The $k$ th singular norm of $\prod$ is defined to be $\left|\prod^{s}\right|_{k}=\sum_{i=1}^{t}\left|\prod_{i}^{s}\right|_{k}$.

\section{ZERO PATTERNS}

A major tool in this section is the following well known Frobenius-König Theorem. The equivalence of Conditions (i) and (iii) is due to König [12, p. 240], [11]. A different proof is suggested by Egerváry [6], who acknowledges the theorem to König. The special case of $m=n=r+1$ was already proven by Frobenius. The 
equivalence of Conditions (i) and (ii) in the theorem is immediate. We add a "furthermore" statement to the theorem which follows easily, and we provide a proof of that statement for the sake of completeness.

THEOREM 3.1 Let $P$ be an $m \times n$ pattern matrix, and let $r$ be a nonnegative integer. Then the following are equivalent:

(i) The smallest number of lines (rows and/or columns) that contain all nonzero elements of $P$ is less than or equal to $r$.

(ii) The pattern matrix $P$ is permutationally equivalent to a pattern

$$
\left(\begin{array}{cc}
P_{11} & P_{12} \\
P_{21} & 0
\end{array}\right), \quad P_{11} \text { is } k \times(r-k), \quad 0 \leq k \leq r .
$$

(iii) $B(P)$ has no $t$-matching for all $t>r$.

Furthermore, if the above conditions hold then every r-matching in $B(P)$ is a union of a $k$-matching in $B\left(P_{12}\right)$ and an $(r-k)$-matching in $B\left(P_{21}\right)$.

Proof We prove the additional statement. Let (ii) hold, and let $M$ be a matching in $B(P)$. Assume that $M$ contains $t$ edges which match rows and columns of $P_{11}$. Observe that then at most $k-t$ rows of $P_{12}$ can match with columns of $P_{12}$, and at most $r-k-t$ rows of $P_{21}$ can match with columns of $P_{21}$. Hence, the cardinality of $M$ cannot exceed $r-t$. It now follows that every $r$-matching in $B(P)$ does not match rows and columns of $P_{11}$, and the claim follows.

We continue with an easy observation.

OBSERVATION 3.3 Let $r$ be a nonnegative integer, and let $A$ be an $m \times n$ matrix over an arbitrary field $\mathbf{F}$ that is permutationally equivalent to a matrix

$$
\left(\begin{array}{cc}
A_{11} & A_{12} \\
A_{21} & 0
\end{array}\right), \quad A_{11} \text { is } k \times(r-k), \quad 0 \leq k \leq r .
$$

Then the following are equivalent:

(i) $A$ is of rank $r$.

(ii) $A_{12}$ is of rank $k$ and $A_{21}$ is of rank $r-k$.

THEOREM 3.4 Let $\mathbf{F}$ be a field of at least three elements. Let $P$ be an $r \times n$ pattern matrix, and assume that $B(P)$ has no constrained $r$-matching. Then there exists a matrix $A$ over $\mathbf{F}$ with pattern $P$ such that the rows of $A$ are linearly dependent.

Proof We prove our assertion by induction is $r$. If $r=1$ then every $r$-matching in $B(P)$ is constrained and there is nothing to prove. Assume our claim holds for $r<p$ where $p>1$, and let $r=p$. Let $A$ be an $r \times n$ matrix with pattern $P$ and such that the nonzero elements of $A$ are algebraically independent indeterminates. If no column of $A$ contains exactly one nonzero indeterminate then, since $\mathbf{F}$ has at least three elements, we can assign the indeterminates in $A$ nonzero values such that the column sums of the resulting matrix $C$ are all zero. This may be done in a simple algorithm that assigns nonzero values to the indeterminates in a column 
such that the proper partial column sums (read from the top) are nonzero and the column sums are zero. Therefore, the sum of the rows of $C$ is zero, and hence the rows of $C$ are linearly dependent. If $A$ has a column $j$ with just one indeterminate in the $(i, j)$ position then, since $B(P)$ does not have a constrained $r$-matching, the bipartite graph of the $(r-1) \times n$ matrix $A(i \mid\langle n\rangle]$ does not have a constrained $(r-1)$-matching. By the inductive assumption, there exists a matrix $C$ with pattern $P$ with a non-trivial linear combination of the rows of $C(i \mid\langle n\rangle]$ that is equal to zero. Since $(i, j)$ is the only nonzero position in the $j$ th column of $A$, it follows that the same linear combination of the rows of $C$ is equal to zero.

COROLlaRY 3.5 Let $\mathbf{F}$ be a field of at least three elements. Let $P$ be an $m \times r$ pattern matrix, and assume that $B(P)$ has no constrained $r$-matching. Then there exists a matrix $A$ over $\mathbf{F}$ with pattern $P$ such that the columns of $A$ are linearly dependent.

We remark that Theorem 3.4 and Corollary 3.5 do not hold in general for a field with two elements, as demonstrated by the following example.

EXAMPLE 3.6 Let $\mathbf{F}$ be the field $\{0,1\}$ and let $E$ be the matrix

$$
\left(\begin{array}{lll}
1 & 1 & 0 \\
0 & 1 & 1 \\
1 & 1 & 1
\end{array}\right)
$$

It is easy to verify that $B(E)$ has no constrained 3-matching. Also the determinant of $E$ is equal to 1 . Since $E$ is the only matrix with the bipartite graph $B(E)$, it follows that there exists no singular matrix $A$ over $\mathbf{F}$ with $B(A)=B(E)$.

LEMMA 3.7 Let $\mathbf{F}$ be a field of at least three elements. Let $P$ be an $n \times n$ pattern matrix, and assume that $B(P)$ has an n-matching. Then there exists a nonsingular matrix $A$ over $\mathbf{F}$ with pattern $P$.

Proof We prove our assertion by induction on $n$. For $n=1$ the result is obvious. Assume our assumption holds for $n<p$ where $p>1$, and let $n=p$. Let $A$ be an $n \times n$ matrix with pattern $P$ such that the nonzero elements of $A$ are independent indeterminates. Given that $B(P)$ has an $n$-matching, let $[1, j]$ be an edge in such a matching. Let $C$ be the $(n-1) \times(n-1)$ submatrix $A(1 \mid j)$. Observe that $B(A(1 \mid j))$ has an ( $\dot{n}-1)$-matching, and by the inductive assumption we can assign the indeterminates in $C$ nonzero values in $\mathbf{F}$ such that the resulting matrix is nonsingular. We now assign the indeterminates in the first row and in the $j$ th column of $A$ the value 1. If the resulting matrix is nonsingular then we are done. If it is singular then we change the value of $a_{1 j}$ from 1 to 2 to obtain a nonsingular matrix.

Lemma 3.7 does not hold in general for a field with two elements, as demonstrated by the following example. 
(i) Find all $r$ such that there exists a matrix $A$ over any field with pattern $P$ and with rank $r$.

(ii) Find the minimal $r$ such that there exists a matrix $A$ over any field with pattern $P$ and with rank $r$.

(iii) Given a field $\mathrm{F}$, find all $r$ such that there exists a matrix $A$ over $\mathrm{F}$ with pattern $P$ and with rank $r$.

(iv) Given a field $\mathrm{F}$, find the minimal $r$ such that there exists a matrix $A$ over any field with pattern $P$ and with rank $r$.

\section{FORMAL NONSINGULARITY OF SQUARE PATTERNS}

We start this section with a definition of two related matrix properties.

\section{DEFINITION 4.1}

(i) $A$ square matrix $A$ is said to have property $C$ if in every row and every column of $A$ there exists just one element such that both this element and its cofactor are nonzero. If $A$ is $a 1 \times 1$ matrix then $A$ has property $C$ if and only if $A$ is a nonzero matrix.

(ii) A square matrix $A$ is said to have property $D$ if it is permutationally equivalent to a matrix $A^{\prime}$ such that the proper principal minors of $A^{\prime}$ are nonzero, and the product of every two complementary non-principal minors of $A^{\prime}$ is zero.

Observe that if a matrix has property $\mathrm{D}$ then it has also property $\mathrm{C}$.

In a paper by Culik [5], the author proves two theorems, characterizing formally nonsingular pattern matrices. These theorems are combined and restated here, using our terminology. In the sequel we shall show that these results are contained in a stronger theorem, which follows from our results.

THEOREM 4.2 Let F be a field of at least three elements, and let $P$ be an $n \times n$ pattern matrix. Then the following are equivalent:

(i) Every matrix over $\mathrm{F}$ with pattern $P$ is nonsingular.

(ii) $B(P)$ has a constrained n-matching.

(iii) Every matrix $A$ over $\mathrm{F}$ with pattern $P$ has property $\mathrm{C}$.

(iv) $P$ is permutationally equivalent to a pattern matrix $P^{\prime}$ for which we have: The proper principal subpatterns of $P^{\prime}$ are all formally nonsingular over F, and every matrix over $\mathrm{F}$ with pattern $P^{\prime}$ has property $\mathrm{D}$.

In [5], the author poses the question whether Condition (iii) in Theorem 4.2 can be weakened to "Some matrix $A$ with pattern $P$ has property $C$ ". The answer to the question is negative, as demonstrated by the following example.

EXAMPLE 4.3 Let $P$ be the pattern

$$
\left(\begin{array}{llll}
* & * & 0 & 0 \\
* & * & * & 0 \\
0 & * & * & * \\
0 & 0 & * & *
\end{array}\right) .
$$


EXAMPLE 3.8 Let $\mathbf{F}$ be the field $\{0,1\}$ and let $E$ be the matrix

$$
\left(\begin{array}{lll}
1 & 1 & 0 \\
0 & 1 & 1 \\
1 & 0 & 1
\end{array}\right) .
$$

It is easy to verify that $B(E)$ has two 3-matchings. Also the determinant of $E$ is equal to 0 . Since $E$ is the only matrix with the bipartite graph $B(E)$, it follows that there exists no nonsingular matrix $A$ over $\mathbf{F}$ with $B(A)=B(E)$.

THEOREM 3.9 Let $\mathbf{F}$ be a field of at least three elements. Let $P$ be an $m \times n$ pattern matrix, and let $r$ be a nonnegative integer. Then the following are equivalent:

(i) Every matrix over $\mathbf{F}$ with pattern $P$ has rank $r$.

(ii) $P$ is permutationally equivalent to a pattern (3.2) where every matrix over $\mathbf{F}$ with pattern $P_{12}$ has rank $k$ and every matrix over $\mathbf{F}$ with pattern $P_{21}$ has rank $r-k$.

(iii) $B(P)$ has no $t$-matching for $t>r$, and there exists at least one constrained $r$-matching in $B(P)$.

(iv) All square subpatterns of $P$ of order greater than $r$ are formally singular and at least one $r \times r$ subpattern of $P$ is formally nonsingular.

Proof (i) $\Rightarrow$ (ii). Assume that every matrix over $\mathbf{F}$ with pattern $P$ has rank $r$. Then it follows that $B(P)$ has an $r$-matching. By Lemma 3.7 it follows that $B(P)$ has no $t$-matching for $t>r$. By Theorem 3.1 it follows that $P$ is permutationally equivalent to a pattern 3.2. Since every matrix over $\mathbf{F}$ with pattern $P$ has rank $r$, it follows from Observation 3.3 that every matrix over $\mathbf{F}$ with pattern $P_{12}$ has rank $k$ and every matrix over $\mathbf{F}$ with pattern $P_{21}$ has rank $r-k$.

(ii) $\Rightarrow$ (iii). If (ii) holds then by Theorem $3.1 B(P)$ has no $t$-matching for $t>r$. Furthermore, every $r$-matching in $B(P)$ is a union of a $k$-matching in $B\left(P_{12}\right)$ and an $(r-k)$-matching in $B\left(P_{21}\right)$. Since every matrix over $\mathbf{F}$ with pattern $P_{12}$ has rank $k$, it follows from Theorem 3.4 that $B\left(P_{12}\right)$ has a constrained $k$-matching. Since every matrix over $\mathbf{F}$ with pattern $P_{21}$ has rank $r-k$, it follows from Corollary 3.5 that $B\left(P_{21}\right)$ has a constrained $(r-k)$-matching. It now follows that $B(P)$ has a constrained $r$-matching.

(iii) $\Rightarrow$ (iv). Since $B(P)$ has no $t$-matching for $t>r$, clearly all square subpatterns of $P$ of order greater than $r$ are formally singular. Given that $B(P)$ has a constrained $r$-matching, clearly the corresponding $r \times r$ subpattern of $P$ is formally nonsingular.

(iv) $\Rightarrow$ (i) is clear.

Remark 3.10 We remark that the equivalent conditions in Theorem 3.9 imply also a stronger version of Condition 3.9.iv, without the restriction to at least three elements in the field.

We conclude this section suggesting problems that, in a sense, generalize the discussion of this section.

PROBLEM 3.11 Given a pattern $P$. 
It is easy to verify that if we assign all the nonzero positions in $P$ the value 1 then we obtain a matrix $A$ for which in every row and every column of $A$ there exists just one element such that both this element and its cofactor are nonzero. However, $B(P)$ does not have a constrained 4-matching. Indeed, the matrix

$$
\left(\begin{array}{llll}
1 & 1 & 0 & 0 \\
1 & 2 & 1 & 0 \\
0 & 1 & 2 & 1 \\
0 & 0 & 1 & 1
\end{array}\right)
$$

has pattern $P$ (and even the same sign pattern as $A$ ) and is singular.

Another question araised in [5] is whether Condition (iv) in Theorem 4.2 can be weakened to " $P$ is permutationally equivalent to a pattern matrix $P$ ' for which we have: The proper principal subpatterns of $P^{\prime}$ are all formally nonsingular, and some matrix with pattern $P^{\prime}$ has property $D^{\prime \prime}$. The answer to this question is positive. In the following theorem we show that even a weaker condition, that is Condition (vi) in Theorem 4.4, is equivalent to the formal nonsingularity of $P$.

THEOREM 4.4 Let F be a field of at least three elements, and let $P$ be an $n \times n$ pattern matrix. Then the following are equivalent:

(i) Every matrix with pattern $P$ is nonsingular.

(ii) $B(P)$ has a constrained n-matching.

(iii) $P$ is permutationally equivalent to a triangular pattern with nonzero diagonal elements.

(iv) Every matrix $A$ with pattern $P$ has property $\mathrm{C}$.

(v) $P$ is permutationally equivalent to a pattern $P^{\prime}$ for which the following holds: For every matrix $A$ with pattern $P^{\prime}$, all principal submatrices of $A$ have property $\mathrm{C}$.

(vi) $P$ is permutationally equivalent to a pattern $P^{\prime}$ for which the following holds: There exists a matrix $A$ with pattern $P^{\prime}$ such that all principal submatrices of $A$ have property $\mathrm{C}$.

Proof (i) $\Rightarrow$ (ii) is easy. It also follows from the equivalence of (i) and (iii) in Theorem 3.9.

(ii) $\Leftrightarrow$ (iii) is classical, e.g. Theorem 2.1 in [2].

(iii) $\Rightarrow$ (iv) is clear.

(iv) $\Rightarrow$ (i). Observe that if a matrix has property $\mathrm{C}$ then it is nonsingular. The implication follows.

(iii) $\Rightarrow$ (v) is clear.

(v) $\Rightarrow$ (vi) is obvious.

(vi) $\Rightarrow(\mathrm{i})$. Let (vi) hold. We prove that $B\left(P^{\prime}\right)$ (and therefore $B(P)$ ) has a constrained $n$-matching by induction on $n$. For $n=1$ the proof is trivial. Assume the claim holds for $n\langle p$ where $p>1$ and let $n=p$. Let $S$ be a proper subset of $\langle n\rangle$. By the inductive assumption $B\left(P^{\prime}[S]\right)$ has a constrained $|S|$-matching. Observe that this matching is the set $M_{s}=\{[i, i]: i \in S\}$. It also follows that $M_{\langle n\rangle}$ is an $n$-matching for 
$B\left(P^{\prime}\right)$. In order to show that $M_{\langle n\rangle}$ is a constrained $n$-matching in $B\left(P^{\prime}\right)$ observe that, since $M_{\langle n-1\rangle}$ is a constrained (n-1)-matching in $B\left(P^{\prime}(n \mid n)\right.$ ), it is enough to show that there exists no $n$-matching in $B\left(P^{\prime}\right)$ with an edge $[i, n], i \neq n$, in it. Assume to the contrary that such a matching exists. If there exist two $n$-matchings which contain $[i, n]$ then there exists a set $S$ of cardinality $k$ less than $n$ such that $i, n \in S$ and such that there exists a $k$-matching in $B(P[S])$ which contains the edge $[i, n]$, e.g. the proof of Lemma 2.1 in [7]. This contradicts the fact that $M_{S}$ is a constrained $k$-matching in $B(P[S])$. Therefore, we have to assume that there exists only one $n$-matchings which contains $[i, n]$. But then it follows that both submatrices $A[i \mid n]$ and $A(i \mid n)$ are nonsingular in every matrix with pattern $P^{\prime}$, which contradicts the fact that $P^{\prime}$ has property $C$. Thus, our assumption that there exists an $n$-matchings which contains $[i, n]$ is false, and hence $M_{\langle n\rangle}$ is a constrained $n$-matching in $B\left(P^{\prime}\right)$.

It would be natural to ask whether Condition (vi) in Theorem 4.4 can weakened to " $P$ is permutationally equivalent to a pattern $P$ ' for which the following holds: There exists a matrix $A$ with pattern $P^{\prime}$ such that all leading principal submatrices of $A$ have property C". The answer to this question is negative, as demonstrated by the following example.

EXAMPLE 4.5 Let $P$ be the pattern

$$
\left(\begin{array}{llll}
* & 0 & 0 & * \\
* & * & 0 & * \\
0 & * & * & 0 \\
0 & * & * & *
\end{array}\right) .
$$

It is easy to verify that if we assign all the nonzero positions in $P$ the value 1 then we obtain a matrix $A$ for which the leading principal submatrices of $A$ have property C. However, $B(P)$ does not have a constrained 4-matching. Indeed, the matrix

$$
\left(\begin{array}{llll}
1 & 0 & 0 & 1 \\
1 & 1 & 0 & 2 \\
0 & 1 & 1 & 0 \\
0 & 2 & 1 & 1
\end{array}\right)
$$

has pattern $P$ (and even the same sign pattern as $A$ ) and is singular.

\section{SIGN PATTERNS}

In this section we use the following result, proven as Remark 1.1 in [10].

THEOREM 5.1 Let $P$ be an $m \times n$ sign pattern. Then the following are eqivalent:

(i) Every matrix with sign pattern $P$ has rank $m$.

(ii) Every nonzero $(+,-, 0)$-row scaling of $P$ has a nonzero unisign column.

The following observation is the analog of Lemma 3.7 in the real case. It can be proved very easily, using continuity arguments. 
OBSERVATION 5.2 Let $P$ be an $n \times n$ sign pattern, and assume that $B(P)$ has an $n$-matching. Then there exists a nonsingular matrix $A$ with sign pattern $P$.

We now come to the main theorem of this section.

THEOREM 5.3 Let $P$ be an $m \times n$ sign pattern, and let $r$ be a nonnegative integer. Then the following are equivalent:

(i) Every matrix with sign pattern $P$ has rank $r$.

(ii) $P$ is permutationally equivalent to a pattern 3.2 where every matrix with sign pattern $P_{12}$ has rank $k$ and every matrix with sign pattern $P_{21}$ has rank $r-k$.

(iii) $P$ is permutationally equivalent to a pattern (3.2) where every nonzero $(+,-, 0)$ row scaling of $P_{12}$ has a unisign column, and every $(+,-, 0)$-column scaling of $P_{21}$ has a unisign row.

Proof (i) $\Rightarrow$ (ii). Since every matrix with the sign pattern $P$ has rank $r$, it follows from Observation 5.2 that $P$ cannot have a $t$-matching for $t>r$. By Theorem 3.1 $P$ is permutationally equivalent to a pattern (3.2). Since every matrix $A$ with sign pattern $P$ is of rank $r$, it follows from Observation 3.3 that every matrix with sign pattern $P_{12}$ has rank $k$ and every matrix with sign pattern $P_{21}$ has rank $r-k$.

(ii) $\Leftrightarrow$ (iii) follows from Theorem 5.1.

(ii) $\Rightarrow$ (i). Let $P$ be permutationally equivalent to a pattern (3.2), where every matrix with sign pattern $P_{12}$ has rank $k$ and every matrix with sign pattern $P_{21}$ has rank $r-k$. By Observation 3.3 it follows that every matrix with sign pattern $P$ has rank at $r$.

In the special case of nonnegative sign patterns, the equivalence (i) $\Leftrightarrow$ (iii) in the following theorem was proven as Lemma 5.2 in [14], which preceded [10]. The equivalence (i) $\Leftrightarrow$ (ii) is in Theorem 5.1. For the sake of completeness, we shall provide here a short proof for the equivalence (ii) $\Leftrightarrow$ (iii).

THEOREM 5.4 Let $P$ be a nonnegative $m \times n$ sign pattern. Then the following are equivalent:

(i) Every matrix with sign pattern $P$ has rank $m$.

(ii) Every nonzero $(+,-, 0)$-row scaling of $P$ has a nonzero unisign column.

(iii) The rows of $P$ are combinatorially independent.

Proof (ii) $\Rightarrow$ (iii). Assume that the rows of $P$ are not combinatorially independent. Then we can find two non-empty disjoint sets of rows that have the same support. We leave the rows of one set as are, we change the signs of the elements in the rows of the other set from + to - , and we make all the other rows of $P$ be zero. Observe that this is a nonzero $(+,-, 0)$-row scaling of $P$ that has no nonzero unisign column.

(iii) $\Rightarrow$ (ii). Assume that we can find a nonzero $(+,-, 0)$-row scaling of $P$ that has no nonzero unisign column. Let $S_{1}$ be the set of the rows that are scaled by + and let $S_{2}$ be the set of the rows that are scaled by -. If one of these sets is empty then it follows that $P$ is all zero, and hence its rows are combinatorially dependent. If none of $S_{1}$ and $S_{2}$ is empty then, since every nonzero column of $P$ contains both 
+'s and -'s, it follows that these disjoint sets are have the same support, and hence the rows of $P$ are combinatorially dependent.

In view of Theorem 5.4, the application of Theorem 5.3 to nonnegative sign patterns yields the following.

THEOREM 5.5 Let $P$ be a nonnegative $m \times n$ sign pattern, and let $r$ be a nonnegative integer. Then the following are equivalent:

(i) Every matrix with sign pattern $P$ has rank $r$.

(ii) $P$ is permutationally equivalent to a pattern (3.2) where every matrix with sign pattern $P_{12}$ has rank $k$ and every matrix with sign pattern $P_{21}$ has rank $r-k$.

(iii) $P$ is permutationally equivalent to a pattern (3.2) where the rows of $P_{12}$ are combinatorially independent and the columns of $P_{21}$ are combinatorially independent.

Motivated by Theorem 3.9, it is natural to ask whether the conditions in Theorem 5.3 and/or Theorem 5.5 yield a condition like Condition (iv) in Theorem 3.9, that is that all square subpatterns of $P$ of order greater than $r$ are formally singular and at least one $r \times r$ subpattern of $P$ is formally nonsingular. Indeed, it is easy to show, using Observation 5.2, that Condition (i) in Theorems 5.3 and 5.5 implies that all square subpatterns of $P$ of order greater than $r$ are formally singular. However, Example 5.6 below shows that, in general, the conditions in Theorem 5.3 and/or Theorem 5.5 do not yield the existence of a formally nonsingular $r \times r$ subpattern of $P$.

It would be also natural to ask whether Condition (iii) in Theorem 5.5 implies that either $P$ has at least $r$ combinatorially independent rows or $P$ has at least $r$ combinatorially independent columns. The answer to this question is negative, as demonstrated by the following example.

EXAMPLE 5.6 Let $B$ be the nonnegative $10 \times 5$ sign pattern of the matrix $A$ considered in the example following Lemma 5.2 in [14], and let $P$ be the nonnegative $15 \times 15$ sign pattern

$$
\left(\begin{array}{cc}
0 & B^{T} \\
B & 0
\end{array}\right) .
$$

Since $B$ has 5 combinatorially independent columns, it follows from Theorem 5.5 that every matrix with pattern $P$ has rank 10 . If $P$ had a $10 \times 10$ formally nonsingular subpattern then, by Lemma 5.2 in [14], it would yield that $P$ has 10 rows which are combinatorially independent. Therefore, $B$ would have at least 5 combinatorially independent rows. Since $B$ is known to have no more than 4 combinatorially independent rows, see [14], it thus follows that $P$ has no $10 \times 10$ formally nonsingular subpattern. Also, we have shown that $P$ has no 10 combinatorially independent rows. Since $P$ is a symmetric pattern, it follows that $P$ has no 10 combinatorially independent columns.

We conclude this section suggesting problems similar to the problems in Problem 3.11.

PRoBlem 5.7 Given a sign pattern $P$. 
(i) Find all $r$ such that there exists a matrix $A$ with sign pattern $P$ and with rank $r$.

(ii) Find the minimal $r$ such that there exists a matrix $A$ with sign pattern $P$ and with rank $r$.

\section{PATTERNS THAT DETERMINE RANKS OF ALL POWERS}

In this section we apply the results of Sections 3 and 5 to the question of characterizing square patterns $P$ such that for every positive integer $k$ and for all matrices $A$ with pattern $P$, the matrices $A^{k}$ have the same rank. This is essentially the problem of characterizing graphs with combinatorially determined elementary divisors. This problem is, in general, open, and has been discussed in a few papers, e.g. [3] and [4]. Here we shall prove an improvement to a result proven in [4], using entirely different techniques. All the graphs discussed in this section are (simple) directed graphs, unless stated else explicitly.

DefinItION 6.1 Let $A$ be an $n \times n$ matrix. The graph $G(A)$ of $A$ is defined to be the directed graph with vertex set $\{1, \ldots, n\}$ and where $(i, j)$ is an arc in $G$ whenever $a_{i j} \neq 0$.

DEFINITION 6.2 Let $G$ be a graph.

(i) $A$ set of $t$ arcs $\left(i_{1}, j_{1}\right), \ldots,\left(i_{t}, j_{t}\right)$ in $G$ is said to be a $t$-matching (between $\left\{i_{1}, \ldots, i_{t}\right\}$ and $\left.\left\{j_{1}, \ldots, j_{t}\right\}\right)$ if $i_{1}, \ldots, i_{t}$ are distinct and $j_{1}, \ldots, j_{t}$ are distinct. Such a t-matching is said to be a constrained t-matching if it is the only t-matching in $G$ between $\left\{i_{1}, \ldots, i_{t}\right\}$ and $\left\{j_{1}, \ldots, j_{t}\right\}$.

(ii) Let $S^{1}, \ldots, S^{k+1}$ be sets of $t$ vertices (each) in $G$. $A$ sequence $\left(M^{1}, \ldots, M^{k}\right)$ of $t$-matchings, such that $M^{i}$ is a t-matching between $S^{i}$ and $S^{i+1}, i \in\langle k\rangle$, is said to be a $(k, t)$-matching between $S^{1}$ and $S^{k+1}$ (through $\left.S^{2}, \ldots, S^{k}\right)$. Such $a(k, t)$-matching is said to be a constrained $(k, t)$-matching if it is the only $(k, t)$-matching in $G$ between $S^{1}$ and $S^{k+1}$. Observe that a $(1, t)$-matching is a $t$-matching.

(iii) Let $M$ be a $(k, t)$-matching between $S^{1}$ and $S^{2}$. Observe that $M$ yields a path from each vertex $i$ in $S^{1}$ to a vertex $j$ in $S^{2}$. We say that $M$ matches $i$ with $j$.

(iv) An arc $(i, j)$ in $G$ is said to be involved in a $(k, t)$-matching $M$ in $G$ if it belongs to at least one of the matchings in the sequence $M$.

Remark 6.3 Let $A$ be an $n \times n$ matrix, and let $S^{1}$ and $S^{2}$ be two subsets of cardinality $m$ of $\langle n\rangle$. A $t$-matching between $S^{1}$ and $S^{2}$ in $B(A)$ is, in fact, a $t$ matching between $S^{1}$ and $S^{2}$ in $G(A)$. Therefore, in the square case, one can state the results of the previous sections for $G(P)$ rather than $B(P)$.

CONVENTION 6.4 For every positive integer and every graph $G$, we say that there exists a $(k, 0)$-matching (between $\varnothing$ and $\varnothing)$ in $G$. This matching is constrained.

We shall use the following consequence of the results in the previous sections. 
THEOREM 6.5 Let $\mathbf{F}$ be a field of at least three elements, let $A$ be an $n \times n$ matrix over $\mathbf{F}$, and let $k$ and $t$ be positive integers. If there exists a constrained $(k, t)$-matching in $G(A)$ then $\operatorname{rank}\left(A^{k}\right) \geq t$. Furthermore, if there exists a constrained $(k, t)$-matching in $G(A)$, and there is no $(k, t+1)$-matching in $G(A)$, then $\operatorname{rank}\left(A^{k}\right)=t$.

Proof Let $\alpha$ and $\beta$ be subsets of $\langle n\rangle$ of cardinality $t$. By the Binet-Cauchy formula, $\operatorname{det}\left(A^{k}[\alpha \mid \beta]\right)$ is equal to

$$
\sum_{\substack{\gamma^{1}, \ldots, \gamma^{k-1} \subseteq\langle n\rangle \\\left|\gamma^{\prime}\right|=t}} \operatorname{det}\left(A\left[\alpha \mid \gamma^{1}\right]\right) \operatorname{det}\left(A\left[\gamma^{1} \mid \gamma^{2}\right]\right) \cdots \cdot \operatorname{det}\left(A\left[\gamma^{k-2} \mid \gamma^{k-1}\right]\right) \operatorname{det}\left(A\left[\gamma^{k-1} \mid \beta\right]\right),
$$

Therefore, if there exists a constrained $(k, t)$-matching between $\alpha$ and $\beta$ in $G$ then it follows, by Theorem 3.9, that $\operatorname{det}\left(A^{k}[\alpha \mid \beta]\right) \neq 0$, and hence $\operatorname{rank}\left(A^{k}\right) \geq t$. Also observe that $\operatorname{det}\left(A^{k}[\alpha \mid \beta]\right) \neq 0$ only if there exists a $(k, t)$-matching between $\alpha$ and $\beta$ in $G$. Hence, if there is no $(k, t+1)$-matching in $G(A)$, then $\operatorname{rank}\left(A^{k}\right) \leq t$. The result follows.

The condition that there exists a constrained $(k, t)$-matching in $G(A)$ is not necessary for $A$ to satisfy $\operatorname{rank}\left(A^{k}\right) \geq t$, as demonstrated by the following example.

EXAMPLE 6.6 Let $G(A)$ be the graph

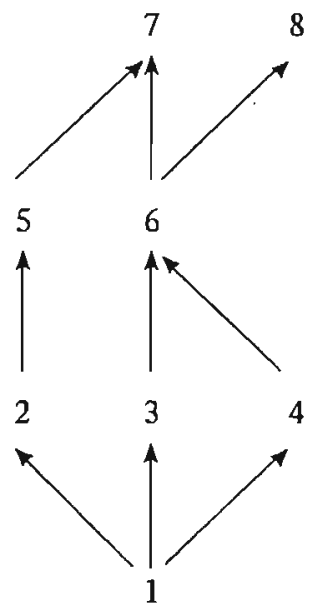

It is easy to verify that there is no 6-matching in $G$ and that there is a constrained 5matching between $\{1,2,3,5,6\}$ and $\{4,5,6,7,8\}$. Therefore, $\operatorname{rank}(A)=5$. Also, there is no (2,4)-matching in $G$ and there is a constrained (2,3)-matching between $\{1,2,3\}$ and $\{5,7,8\}$ (through $\{2,5,6\})$. Therefore, $\operatorname{rank}\left(A^{2}\right)=3$. The only possible nonzero row in $A^{3}$ is the first row. Here we have $\left(A^{3}\right)_{17}=a_{12} a_{25} a_{57}+\left(a_{13} a_{36}+a_{14} a_{46}\right) a_{67}$ and $\left(A^{3}\right)_{18}=\left(a_{13} a_{36}+a_{14} a_{46}\right) a_{68}$. Notice that we cannot have $\left(A^{3}\right)_{17}=\left(A^{3}\right)_{18}=0$, and hence $\operatorname{rank}\left(A^{3}\right)=1$. However, there is no constrained $(3,1)$-matching in $G$. 


\section{DEFINITION 6.7}

(i) A directed graph is said to be triangular if it contains no cycles except possibly for loops.

(ii) $A$ directed graph $G$ is said to be strongly triangular if the undirected graph obtained by removing the directions from the arcs of $G$ contains no cycles except possibly for loops. Observe that a strongly triangular directed graph is triangular.

In the following results we assume that $G$ is a triangular graph, and that $S$ is the set of singular vertices in $G$.

THEOREM 6.8 Let $G$ be a graph consisting of one path $\pi$ with $n$ vertices and possible loops on vertices of $\pi$. Then for every positive integer $k$ there exists a constrained $\left(k, n-\left|\pi^{s}\right| k\right)$-matching in $G$.

Proof Without loss of generality, let $\pi$ be the path $\{1, \ldots, n\}$. We distinguish between two cases:

CASE $1\left|\pi^{s}\right|>k$. In this case we have $\left|\pi^{s}\right| k=k$. Since clearly $n \geq\left|\pi^{s}\right|>k$, we can define the $k+1(n-k)$-sets $S^{i}=\{i, i+1, \ldots, i+n-k-1\}, i \in\langle k+1\rangle$. Since there is a unique path of length $k$ from $i$ to $i+k-1$, and since there is no path of length $k$ from $i$ to $j$ when $j>i+k-1$, it follows that there is a constrained $\left(k, n-\left|\pi^{S}\right|_{k}\right)$-matching between $S^{1}$ and $S^{k+1}\left(\right.$ through $\left.S^{2}, \ldots, S^{k}\right)$.

CASE $2\left|\pi^{S}\right| \leq k$. Here we take $S^{1}=\cdots=S^{k+1}$ to be the set of nonsingular vertices in $G$. If $S^{1}$ is empty then $n-\left|\pi^{S}\right|_{k}=0$ and, in view of Convention 6.4, we are done. Else, since a path in $G$ is a non-decreasing sequence, it follows that every vertex $i$ in $S^{1}$ is matched with itself. Since the only path from $i$ to itself is $(i, i, \ldots, i)$, it follows that there is a constrained $\left(k, n-\left|\pi^{S}\right|_{k}\right)$-matching between $S^{1}$ and $S^{k+1}$ (through $S^{2}, \ldots, S^{k}$ ).

Let $G$ be a graph consisting of one path $\pi$ with $n$ vertices and possible loops on vertices of $\pi$. In view of Theorem 6.8 , it is natural to ask whether every $(k, m)$ matching in $G$ is constrained. The answer to this question is negative, as demonstrated by the following example.

EXAMPLE 6.9 Let $G$ consist of the path $(1,2,3,4)$ with loops on 2,3 and 4 . Observe that the paths $(1,2,2,3)$ and $(2,3,3,4)$, as well as the paths $(1,2,3,3)$ and $(2,3,4,4)$, form $(3,2)$-matchings between $(1,2)$ and $(3,4)$.

As an immediate corollary of Theorems 6.5 and 6.8 we now obtain

COROLLARY 6.10 Let $G$ be a graph consisting of one path $\pi$ with $n$ vertices and possible loops on vertices of $\pi$, and let $\mathrm{F}$ be a field of at least three elements. Then for every positive integer $k$ and for all matrices $A$ over $\mathbf{F}$ with $G(A)=G$ we have $\operatorname{rank}\left(A^{k}\right) \geq n-\left|\pi^{S}\right|_{k}$.

THEOREM 6.11 Let $G$ be a strongly triangular graph, let $S^{1}$ and $S^{2}$ be sets of $t$ vertices in $G$, and let $(i, j), i \neq j$, be an arc that is involved in a $(k, t)$-matching between $S^{1}$ and $S^{2}$ for some positive integer $k$. Let $m$ be a positive integer, and let $M$ be an $(m, t)$-matching between $S^{1}$ and $S^{2}$. Then $(i, j)$ is involved in $M$. 
Proof Let $M^{1}$ and $M^{2}$ be $(k, t)$-matching and $(m, t)$-matching respectively between $S^{1}$ and $S^{2}$. We color the arcs on the paths from vertices in $S^{1}$ to vertices in $S^{2}$ yielded by $M^{1}$ with blue, while we color the arcs on the paths yielded by $M^{2}$ with red. Observe that an arc may have two colors. For a vertex $i$ in $S^{1}$ we move along the blue path from $i$ to the vertex $j$ in $S^{2}$ that is matched with $i$ by $M^{1}$. We then move backwards along the red path to the vertex $i^{\prime}$ in $S^{1}$ that is matched with $j$ by $M^{2}$. We continue this way until we get back to $i$ in $S^{1}$. As we follow this procedure for every vertex $i$ in $S^{1}$, we cover all the arcs involved in $M^{1}$ and $M^{2}$. Since each such a "walk" terminates in its starting point, and since $G$ is strongly triangular, it follows that if move forwards along an $\operatorname{arc}(i, j), i \neq j$, then in some stage we also have to move backwards along that arc. Therefore, each colored arc $(i, j), i \neq j$, is colored both blue and red, and our claim follows.

THEOREM 6.12 Let $G$ be a strongly triangular graph, let $\Pi$ be a union of disjoint paths in $G$, and let $n$ be the number of vertices covered by the paths in $\Pi$. Then there exists a constrained $\left(k, n-\left|\Pi^{S}\right|_{k}\right)$-matching in $G$.

Proof By Theorem 6.8, there exists a constrained $\left(k,\left|\pi_{i}\right|-\left|\pi_{i}^{S}\right|_{k}\right)$-matching $M_{i}$ between some sets $S_{i}^{1}$ and $S_{i}^{2}$ in $\pi_{i}$. The union of these matchings is a $\left(k, n-\left|\prod^{S}\right|_{k}\right)$ matching $M^{1}$ between sets $S^{1}$ and $S^{2}$ in the union $G^{\prime}$ of the disjoint paths in $\Pi$. Let $M^{2}$ be any $\left(k, n-\left|\Pi^{S}\right|_{k}\right)$-matching between $S^{1}$ and $S^{2}$ in $G$. Since $G$ is strongly triangular, it follows by Theorem 6.11 that $M^{2}$ is a matching in $G^{\prime}$, and hence $M^{2}$ is a union of $\left(k,\left|\prod_{i}\right|-\left|\pi_{i}^{S}\right|_{k}\right)$-matchings between the sets $S_{i}^{1}$ and $S_{i}^{2}$ in $\pi_{i}$. Since $M_{i}$ is a constrained such matching, it follows that $M^{2}$ is the same as $M^{1}$.

We can now prove the main result of this section.

THEOREM 6.13 Let $P$ be an $n \times n$ pattern matrix such that $G(P)$ is strongly triangular, and let $\mathrm{F}$ be a field of at least three elements. Let $k$ be a positive integer. Then for all matrices $A$ over $\mathrm{F}$ with pattern $P$, the matrices $A^{k}$ have the same rank.

Proof Let $\Pi$ be a path covering for $S$ such that $\left|\Pi^{S}\right|_{k}$ is minimal. By Theorems 6.5 and 6.12, we have $\operatorname{rank}\left(A^{k}\right) \geq n-\left|\Pi^{S}\right|_{k}$. By Theorems 5.6 and 5.11 in [9] we have $\operatorname{rank}\left(A^{k}\right) \leq n-\left|\Pi^{S}\right|_{k}$. Therefore, we have $\operatorname{rank}\left(A^{k}\right)=n-\left|\Pi^{S}\right|_{k}$, and the assertion follows.

Remark 6.14 Theorem 6.13 improves Theorem 2.4 in [4]. It can easily be shown that if the graph $D_{0}(A)$ as defined in [4] is a directed forest (see definitions in [4]), then $G(A)$ is strongly triangular. Therefore, if $D_{0}(A)$ is a directed forest then it follows from Theorem 6.13 that the elementary divisors of $A$ are combinatorially determined, as shown in Theorem 2.4 in [4]. However, if $G(A)$ is strongly triangular then $D_{0}(A)$ is not necessarily a directed forest, as demonstrated by Example 4.7 in [9].

Denote by $n(A)$ the nullity of a square matrix $A$. Recall that the height characteristic of a square matrix $A$ is defined to be the sequence $\left(\eta_{1}, \ldots, \eta_{p}\right)$, where $\eta_{i}=n\left(A^{i}\right)-n\left(A^{i-1}\right), i \in\langle p\rangle\left(n\left(A^{0}\right)=0\right)$, and where $p$ is the index of $A$, that is the minimal positive integer such that $n\left(A^{p}\right)=n\left(A^{p+1}\right)$. Using these terms, Theorem 6.13 can be restated as follows. 
THEOREM 6.15 Let $P$ be an $n \times n$ pattern matrix such that $G(P)$ is strongly triangular, and let $\mathbf{F}$ be a field of at least three elements. Then all matrices over $\mathbf{F}$ with pattern $P$ have the same height characteristic.

According to our results, in order to evaluate the height characteristic in Theorem 6.16 one has to find the minimal $k$ th singular norms of path coverings for $S$. This also follows from the results in [8] and [9]. As observed in those papers, one can alternatively take the dual of the sequence of differences of the maximal cardinalities of sets of singular vertices that can be covered by $k$ disjoint paths.

We conclude the paper by remarking that the condition that $G(P)$ is strongly triangular is not necessary for that all matrices with pattern $P$ to have the same height characteristic, as demonstrated by Example 6.6. All matrices with that graph have height characteristic $(3,2,2,1)$, however the graph is not strongly triangular.

\section{ACKNOWLEDGMENT}

The authors are grateful to Judith McDonald and Chun-Hui Chen for reading the paper and making useful comments. We also thank Professor Bit-Shun Tam for helpful comments.

\section{References}

[1] L. Bassett, J. Maybee and J. Quirk, Qualitative economics and the scope of the correspondence principle, Econometrica 26 (1968), 544-563.

[2] R. A. Brualdi, Permanent of the direct product of matrices, Pacific J. Math. 16 (1966), 471-482.

[3] R. A. Brualdi, Combinatorial verification of the elementary divisors of tensor products, Linear Algebra Appl. 71 (1985), 31-47.

[4] R A. Brualdi, Combinatorially determined elementary divisors, Congr. Numer. 58 (1987), $193-216$.

[5] K. Culik, Absolute rank of square matrices (Russian), Časopis Pěst. Mat. 85 (1960), 457-464.

[6] E. Egerváry, Mátrixok kombinatórikus tulajdonságairól, Matematikai és Fizikai Lapok 38 (1931), 16-28.

[7] G. M. Engel and H. Schneider, The Hadamard-Fischer inequality for a class of matrices defined by eigenvalue monotonicity, Linear and Multilinear Algebra 4 (1976), 155-176.

[8] E. R. Gansner, Acyclic digraphs, Young tableaux, and nilpotent matrices, SLAM J. Algebra Disc. Methods 2 (1981), 429-440.

[9] D. Hershkowitz and H. Schneider, Path coverings of graphs and height characteristics of matrices, J. Combinatorial Theory, Series $B$ (to appear).

[10] V. Klee, R. Ladner and R. Manber, Signsolvability revisited, Linear Algebra Appl. 59 (1984), 131157.

[11] D. König, Graphok és matrixok, Matematikai és Fizikai Lapok 38 (1931), 116-119.

[12] D. König, Theorie der endlichen und unendlichen Graphen, Akad. Verlagsges. 1936, republished by Chelsea 1950.

[13] J. Maybee and J. Quirk, Qualitative problems in matrix theory, SLAM Rev. 11 (1969), 30-51.

[14] D. J. Richman and H. Schneider, On the singular graph and the Weyr characteristic of and $M$-matrix, Aequationes Math. 17 (1978), 208-234. 